\title{
Correlación entre medidas antropométricas y peneanas en la población mexicana
}

\section{Correlation of anthropometric and penile measurements in a Mexican population}

Luis Daniel Carrillo-Córdova, Daniel Calvo-Mena, Eli Jalil Camilo-Martínez, Noé Garduño-Segovia, Samuel Aguilar-Pérez, Mateo Leopoldo Garduño-Arteaga, Jorge Jaspersen-Gastelum

\begin{abstract}
Resumen
OBJETIVO: Correlacionar las dimensiones del pene en flacidez (longitud y diámetro) con medidas antropométricas en la población mexicana adulta.

MATERIALES Y MÉTODOS: Estudio transversal efectuado en hombres que acudieron al servicio de Urología del Hospital General de México, entre marzo y octubre de 2017. Se determinaron las medidas de circunferencia de la base del pene, circunferencia del surco balano-prepucial; longitud de la base a la punta y longitud de la base a la punta en estiramiento; además, se buscaron correlaciones entre éstas y datos antropométricos como: talla, peso, índice de masa corporal e índice cintura-cadera.

RESULTADOS: Se registraron 150 pacientes. La talla media fue de $1.65 \mathrm{~m}( \pm 0.76)$, IMC $27 \mathrm{~kg} / \mathrm{m}^{2}( \pm 4.3)$ e índice cintura-cadera $1.02( \pm 0.133)$. En cuanto a las medidas peneanas: longitud de la base a la punta $8.7 \mathrm{~cm}( \pm 1.8)$, longitud de la base a la punta en estiramiento $10.9( \pm 2.2)$, circunferencia de la base del pene $8.1 \mathrm{~cm}( \pm 1.3)$, circunferencia del surco balano-prepucial $8.4 \mathrm{~cm}( \pm 1.3)$ y pliegue pubiano $2.7( \pm 2.1)$. La longitud de la base a la punta en estiramiento tuvo correlación lineal negativa con el IMC, índice cintura-cadera y peso $(r=-0.282, p<0.01, r=-0.221, p<0.05, r=-0.204$, $\mathrm{p}<0.01)$. Además, se encontró correlación lineal positiva de la longitud de la base a la punta con la circunferencia del surco balano-prepucial y la circunferencia de la base del pene ( $r=0.596, p<0.01$ y $0.522, p<0.01$, respectivamente).
\end{abstract}

CONCLUSION: El tamaño del pene en la población mexicana es similar al reportado en otras series internacionales.

PALABRAS CLAVE: Tamaño del pene; longitud del pene; grosor del pene.

\section{Abstract}

OBJECTIVE: To correlate the measurements of the flaccid penis (length and diameter) with anthropometric measurements in an adult Mexican population.

MATERIALS AND METHODS: A cross-sectional study was conducted on men that sought medical attention at the Urology Service of the Hospital General de México between March and October 2017. Penile base circumference (PBC), balanopreputial sulcus circumference (BPSC), length from the base to the tip in the flaccid penis (LF), and length from the base to the tip in the stretched penis (LS) were measured. Correlations between those measurements and anthropometric data, such as height, weight, body mass index (BMI), and waist-to-hip ratio, were evaluated.

RESULTS: The study included 150 men. Mean height was $1.65 \mathrm{~m}( \pm 0.76), \mathrm{BMI} 27 \mathrm{~kg} /$ $\mathrm{m}^{2}( \pm 4.3)$, and waist-to-hip ratio $1.02( \pm 0.133)$. Mean penile measurements were: LF $8.7 \mathrm{~cm}( \pm 1.8)$, LS $10.9( \pm 2.2)$, PBC $8.1 \mathrm{~cm}( \pm 1.3)$, BPSC $8.4 \mathrm{~cm}( \pm 1.3)$, and the pubic fold $2.7( \pm 2.1)$. There was a negative linear correlation between the LS and BMI, WHR, and weight $(r=-0.282, p<0.01, r=-0.221, p<0.05, r=-0.204, p<0.01$, respectively) and a positive linear correlation between the LF and the BPSC and PBC $(r=0.596, p<0.01$ and $0.522, p<0.01$, respectively).

CONCLUSIONS: Penis size in the Mexican population studied was similar to that reported in other international case series.

KEYWORDS: Size of the penis; Penile length; Penile girth.
Servicio de Urología, Hospital Genera de México Dr. Eduardo Liceaga, Ciudad de México.

Recibido: noviembre 2017

Aceptado: junio 2018

Correspondencia

Luis Daniel Carrillo Córdova carrillocor@gmail.com

Este artículo debe citarse como Carrillo-Córdova LD, Calvo-Mena D, Camilo-Martínez EJ, Garduño-Segovia N, Aguilar-Pérez S, Garduño-Arteaga ML, Jaspersen-Gastelum J. Correlación entre medidas antropométricas y peneanas en la población mexicana. Rev Mex Urol. 2018 julio-agosto; 78(4):254-262. DOI: https://doi.org/10.24245/revmexurol.v78i4.1812 


\section{ANTECEDENTES}

En la actualidad el servicio de Urología suele atender consultas relacionadas con el tamaño del pene. Esto obedece, en buena medida, a circunstancias socioculturales e históricas. El pene se asocia con la idea de fertilidad y masculinidad, además de su relación con la forma, tamaño y longitud. Un ejemplo histórico es el dios griego Príapo de la fertilidad o la clasificación genital de la India por Vatsyayana en el Kama Sutra $(400 \mathrm{aC}) .^{1,2}$ Estos preceptos culturales Ilevaron a que estudios previos estimaran que $55 \%$ de los hombres se encuentran satisfechos con el tamaño de su pene y $45 \%$ desearía tener mayor tamaño. Lo anterior deriva en problemas psicológicos, como el síndrome de ansiedad del pene pequeño y dismorfofobia peneana, ${ }^{3,4}$ cuya consecuencia es el aislamiento, escaso contacto sexual, angustia y excesiva autoconsciencia de la forma y tamaño del pene. ${ }^{4}$

Desde el decenio de 1940 iniciaron las publicaciones relacionadas con las dimensiones peneanas y se estableció la definición de "micropene", como el órgano menor de $7 \mathrm{~cm}$ en erección y menor de $4 \mathrm{~cm}$ en flacidez. ${ }^{5,6}$ Kinsey clasifica al pene en forma, tamaño y angulación, y reporta como promedios normales: angulación de $60-85^{\circ}$ de la horizontal y longitud de 15.24 $\mathrm{cm} .7,8$

La medición del pene es imperativa, pues permite ofrecer ayuda a los varones que lo refieren pequeño, además de utilizarla para fines académicos y didácticos. Hasta la fecha existen cuatro estudios que han producido un nomograma en sus muestras para tratar de resolver esta interrogante; ${ }^{9}$ sin embargo, no existe una revisión sistemática relacionada con el tamaño del pene. El último nomograma realizado incluye 21 estudios, de 1985 a 2014, en diferentes países, pero no toma en cuenta ningún estudio efectuado en México ni América Latina; por tanto, no pueden extrapolarse a nuestro medio y la mayor parte de los estudios no señalan medidas antropométricas asociadas con el tamaño del pene. ${ }^{10,11}$ En América Latina solo existe un estudio publicado en Guatemala respecto del tamaño del pene. ${ }^{12}$

Por lo anterior, el objetivo primario de este estudio fue: correlacionar las dimensiones del pene en flacidez (longitud y diámetro) con el peso, talla, índice de masa corporal (IMC) e índice cintura-cadera en la población mexicana adulta. Como objetivos secundarios se pretendió establecer si existe satisfacción del paciente con el tamaño de su pene y estimar la incidencia de disfunción eréctil entre la población.

\section{MATERIALES Y MÉTODOS}

Estudio transversal, llevado a cabo en la consulta externa del servicio de Urología del Hospital General de México, entre marzo y octubre de 2017. Se excluyeron a los pacientes con diagnóstico de patología peneana o testicular, definidos como: enfermedad de Peyronie, curvatura peneana, estenosis de uretra, tumor testicular, hidrocele, varicocele, antecedente de orquidopexia u atrofia testicular.

Las mediciones las tomaron dos urólogos adiestrados en la técnica de medición para reducir el error interobservador y en condiciones ambientales similares para evitar la variabilidad natural del tamaño, debido a factores temporales como: hora del día y temperatura ambiente. Las mediciones del pene se efectuaron entre las 10:00 am y 16:00 pm, con luz y temperatura ambientales, con los sujetos de pie y con el pene paralelo al piso. La longitud se midió con una cinta métrica, con marcas milimétricas a lo largo del dorso, desde la unión pubopeneana hasta la punta del glande (meato), con extensión máxima, pero no dolorosa. La medición se realizó durante los estados flácido y estirado. El diámetro se midió 
a partir de la base del pene, de la unión pubopeneana y del surco balanoprepucial.

Se recabaron datos demográficos y antropométricos como: edad, talla, peso, antecedente de circuncisión; antecedente de diabetes mellitus, hipertensión arterial u otras comorbilidades. Las mediciones se llevaron a cabo con cintas métricas estandarizadas idénticas y de la misma marca; se efectuó la medición de la circunferencia abdominal, de la cadera y torácica; longitud de la brazada y de hombro a hombro. En cuanto a los parámetros del pene en reposo: circunferencia de la base del pene (CBP), circunferencia del surco balano-prepucial (CP), longitud de la base a la punta (LR) y longitud de la base a la punta en estiramiento (LE). Para la medición del pliegue pubiano se utilizó un plicómetro.

Se documentó la satisfacción del paciente con el tamaño de su pene de manera categórica, con un resultado dicotómico (Sí o No), y se aplicó el Cuestionario de Disfunción Eréctil IIEF-5. El diagnóstico nutricional se estableció con puntos de cortes estandarizados para IMC, considerando $18.5-24.9 \mathrm{~kg} / \mathrm{m}^{2}$ como peso normal, $\geq 25-29.9$ $\mathrm{kg} / \mathrm{m}^{2}$ sobrepeso y $\geq 30 \mathrm{~kg} / \mathrm{m}^{2}$ obesidad.

\section{Análisis estadístico}

Se realizó estadística descriptiva con medidas de tendencia central y dispersión; las variables categóricas se expresaron como medidas de frecuencia absoluta y relativa, y las lineales como media y desviación estándar (DE), o mediana y rangos intercuartilares (RIQ), según correspondieran con la distribución de frecuencias. Las mediciones peneanas se dividieron en percentiles y la talla en tertiles.

La estadística inferencial incluyó pruebas de hipótesis para evaluar las variables lineales como: $t$ de Student para muestras relacionadas y ANOVA para la comparación de medias de las medidas peneanas entre grupos de talla estratificados por tertiles. Las variables categóricas se analizaron con las pruebas de $\chi^{2}$ o exacta de Fisher.

Se utilizó la correlación de Pearson para medidas peneanas y antropométricas. Además, se efectuó la concordancia de kappa para pacientes inconformes con el tamaño de su pene y pertenecer al percentil 10 de las mediciones: longitud de la base a la punta en estiramiento (LE) y longitud de la base a la punta (LR).

Todas las pruebas de hipótesis tuvieron un error alfa ajustado para $\mathrm{p}<0.05$. El análisis de los datos se llevó a cabo con el programa STATASE 13.1.

\section{RESULTADOS}

Se incluyeron 150 pacientes, cuyas características demográficas y antecedentes médicos se muestran en el Cuadro 1. La talla media fue de $1.65 \mathrm{~m}$ ( \pm 0.76$), \mathrm{IMC}$ de $27 \mathrm{~kg} / \mathrm{m}^{2}( \pm 4.3)$; circunferencia torácica de $96.5 \mathrm{~cm}( \pm 9)$ y abdominal

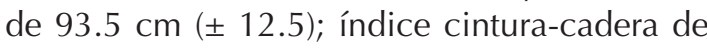

Cuadro 1. Características generales y medición peneana $(\mathrm{n}=150)$

\begin{tabular}{|l|l|}
\hline Demográficas & \\
\hline Edad en años, media (DE) & $56.8(17.4)$ \\
\hline Antropométricas & \\
\hline Peso (kg), media (DE) & $73.8(13.1)$ \\
\hline Talla (m), media (DE) & $1.65(0.76)$ \\
\hline Sobrepeso & $65(43.3 \%)$ \\
\hline Obesidad & $34(22.7 \%)$ \\
\hline Diabetes mellitus tipo 2 & $19(12.7 \%)$ \\
\hline Hipertensión arterial sistémica & $40(26.7 \%)$ \\
\hline Circuncisión & $17(11.3 \%)$ \\
\hline Disfunción eréctil & $90(60 \%)$ \\
\hline
\end{tabular}


$1.02( \pm 0.133)$, longitud de la brazada $165 \mathrm{~cm}$ ( \pm 14.1 y medición hombro a hombro de 42.5 $\mathrm{cm}( \pm 3.8)$. En cuanto a las medidas peneanas: longitud de la base a la punta $8.7 \mathrm{~cm}( \pm 1.8)$, longitud de la base a la punta en estiramiento $10.9( \pm 2.2)$; circunferencia de la base del pene $8.1 \mathrm{~cm}( \pm 1.3)$ y del surco balano-prepucial 8.4 $\mathrm{cm}( \pm 1.3)$, y medición del pliegue pubiano 2.7 $\mathrm{cm}( \pm 2.1)$. Al aplicar el Cuestionario IIEF-5 se encontraron $40 \%$ de pacientes sin disfunción eréctil, 30\% con disfunción eréctil leve, 20\% leve-moderada, $10 \%$ moderada y $10 \%$ severa.

\section{Relación entre medidas antropométricas y peneanas}

La población de estudio se dividió por tertiles de talla con los siguientes puntos de corte: para $\mathrm{T} 1<1.62 \mathrm{~m}, \mathrm{~T} 2=1.62-1.69 \mathrm{~m}$ y $\mathrm{T} 3>169$ m (Cuadro 2). Posteriormente se comparó la media de longitud peneana, observando que las medidas fueran similares entre los diversos grupos de talla, pero con disminución del gradiente de media en la variable de longitud de la base a la punta en estiramiento conforme al IMC (Figura 1). La longitud de la base a la punta del pene en estiramiento mostró una correlación lineal negativa con el IMC, índice cintura-cadera y peso $(r=-0.282, p<0.01 ; r=$ $-0.221, p<0.05 ; r=-0.204, p<0.01$ ) (Figura 2). Además, se encontró una correlación lineal positiva de la longitud de la base a la punta con la circunferencia del surco balano-prepucial y de la circunferencia de la base del pene $(r=$ $0.596, p<0.01$ y $0.522, p<0.01$, respectivamente) (Figura 3). El resto de las correlaciones se muestra a detalle en el Cuadro 3.

\section{Satisfacción del paciente con el tamaño del} pene

Al dividir a la población por percentiles de tamaño del pene se encontraron puntos de corte en el percentil 10: p10 de longitud de la base a
Cuadro 2. Comparación de medidas antropométricas y peneanas (pacientes divididos por tertiles de talla en metros)

\begin{tabular}{|l|c|c|c|c|}
\hline Medidas & T1 & T2 & T3 & p \\
\hline Talla & $1.62 \mathrm{~m}$ & $\begin{array}{c}1.62- \\
1.69\end{array}$ & $>1.60 \mathrm{~m}$ & $\mathrm{p}^{*}$ \\
\hline IMC $\left(\mathrm{kg} / \mathrm{m}^{2}\right)$ & $26(4.7)$ & $28(4.5)$ & $26(3.7)$ & 0.052 \\
\hline ICC & $1.01(0.10)$ & $\begin{array}{c}1.02 \\
(0.11)\end{array}$ & $\begin{array}{c}1.01 \\
(0.15)\end{array}$ & 0.728 \\
\hline CA $(\mathrm{cm})$ & $91(12)$ & $94(10)$ & $93(14)$ & 0.521 \\
\hline CT $(\mathrm{cm})$ & $95(8.5)$ & $97(9.2)$ & $96(9.1)$ & 0.318 \\
\hline CBP $(\mathrm{cm})$ & $7.7(1.1)$ & 8.0 & $8.4(1.2)$ & 0.025 \\
\hline CP $(\mathrm{cm})$ & $8.1(1.1)$ & 8.3 & $8.6(1.3)$ & 0.135 \\
\hline LR $(\mathrm{cm})$ & $8.6(1.9)$ & 8.6 & $8.9(1.8)$ & 0.571 \\
\hline LE $(\mathrm{cm})$ & $10.8(2.3)$ & $10.5(2)$ & 11.3 & 0.228 \\
\hline Pliegue & $2.8(2.5)$ & $3(2.5)$ & $2.2(1)$ & 0.207 \\
pubiano $(\mathrm{cm})$ & & & & \\
\hline
\end{tabular}

* Prueba para ANOVA entre los grupos. Datos expresados como media (DE).

IMC: índice de masa corporal, ICC: índice cintura-cadera, CA: circunferencia abdominal, CT: circunferencia de tórax, CBP: circunferencia de la base del pene, CP: circunferencia del surco balano-prepucial, LR: longitud de la base a la punta, LE: longitud de la base a la punta en estiramiento.

la punta de $<6.82 \mathrm{~cm}$ y $\mathrm{p} 10$ de la longitud de la base a la punta en estiramiento de $<8 \mathrm{~cm}$. En cuanto a la satisfacción con el tamaño del pene, 22 de 150 pacientes refirieron inconformidad. Se observó coincidencia leve entre pertenecer al p10 de tamaño del pene y estar inconforme con su longitud, para las variables de longitud de la base a la punta y longitud de la base a la punta en estiramiento $(\kappa=0.172, p=0.047$ y $\kappa$ $=0.294, p=0.002$, respectivamente).

\section{DISCUSIÓN}

Este es uno de los primeros estudios en la población mexicana que establece la correlación entre medidas peneanas, medidas antropométricas y satisfacción del paciente con el tamaño del pene. 


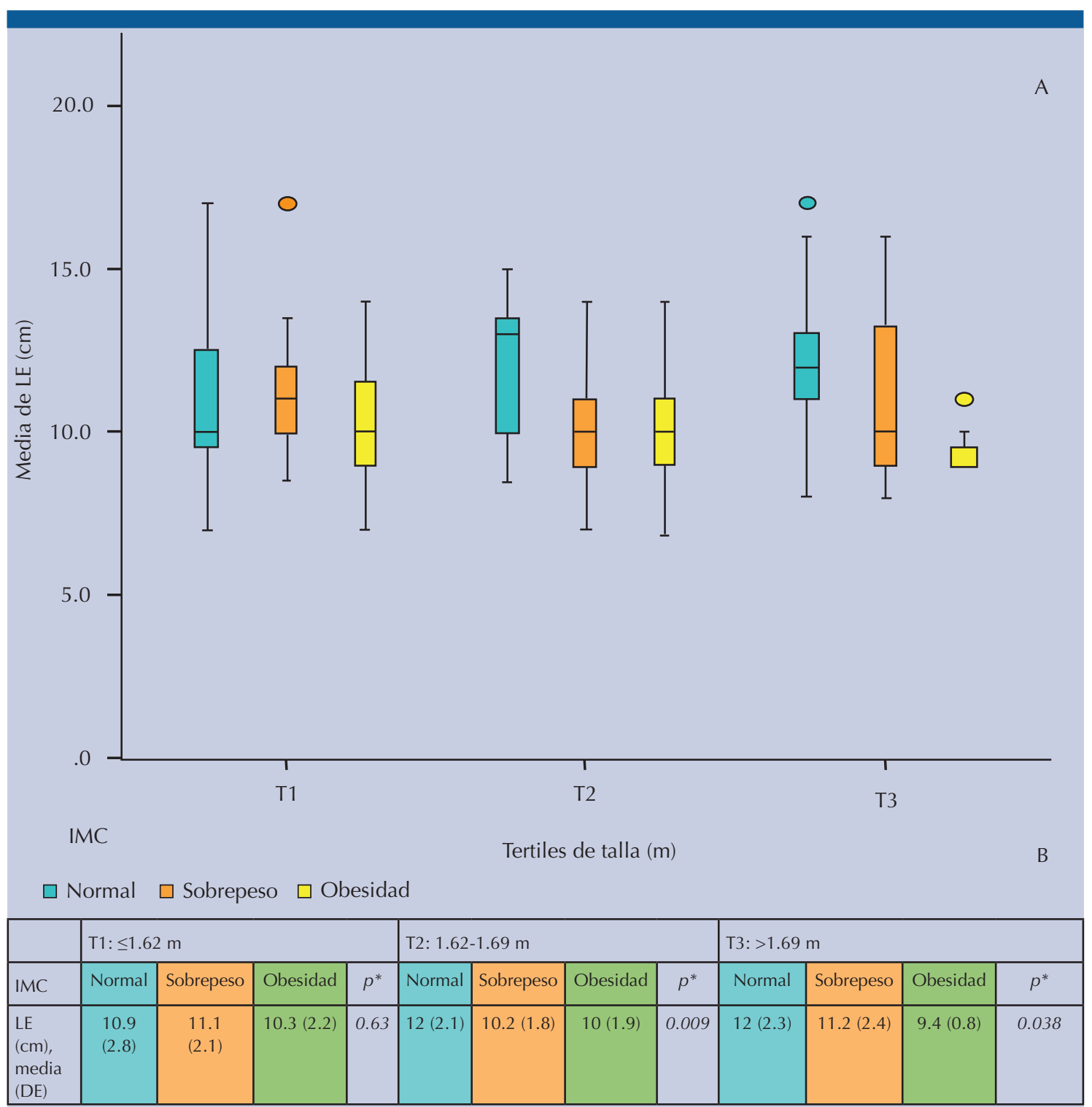

*Prueba F para comparación de grupos.

Comparación de medias de longitud peneana en estiramiento según estiramiento y grupo IMC.

Abreviaturas: T: teril IMC: índice de masa corporal, LE: longitud peneana de estiramiento, DE: desviación estándar.

Figura 1. Comparación de medias de longitud peneana en estiramiento en pacientes mexicanos según los tertiles de estatura, estratificado por IMC (A). No existe diferencia entre la longitud y la estatura; sin embargo, cada grupo muestra un gradiente de diferencia de media conforme aumenta el IMC (B).

Una serie de 3300 adultos jóvenes italianos reportó una longitud promedio del pene en flacidez de $9 \mathrm{~cm}$ y diámetro de $10 \mathrm{~cm} \cdot .^{13}$ Otra investigación en la población Hindú, que estudió a 301 pacientes, señaló una longitud en flacidez de $8.2 \mathrm{~cm}$ y circunferencia de 9.14 


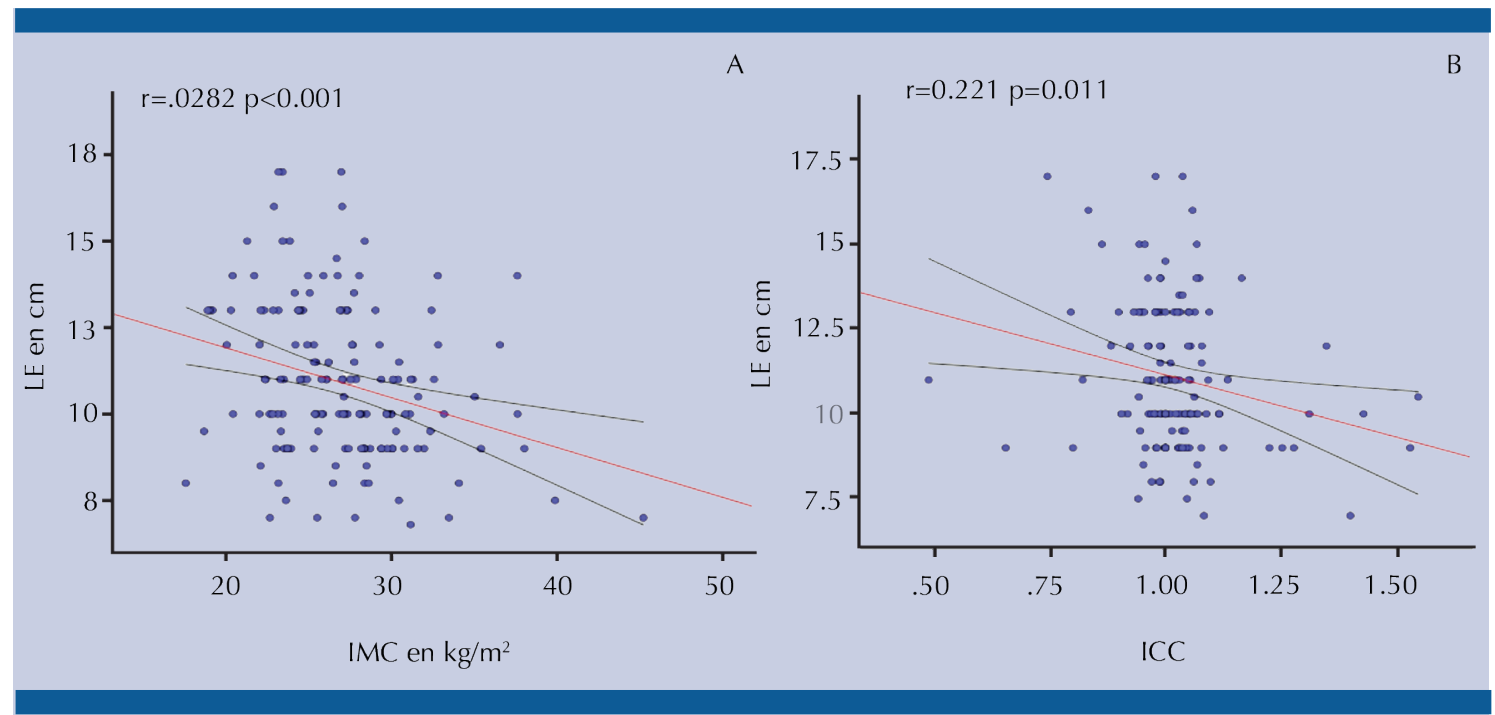

Figura 2. Correlación lineal negativa de la longitud del pene con las medidas antropométricas. IMC: índice de masa corporal; ICC: índice cintura-cadera; LE: longitud de la base a la punta del pene en estiramiento.

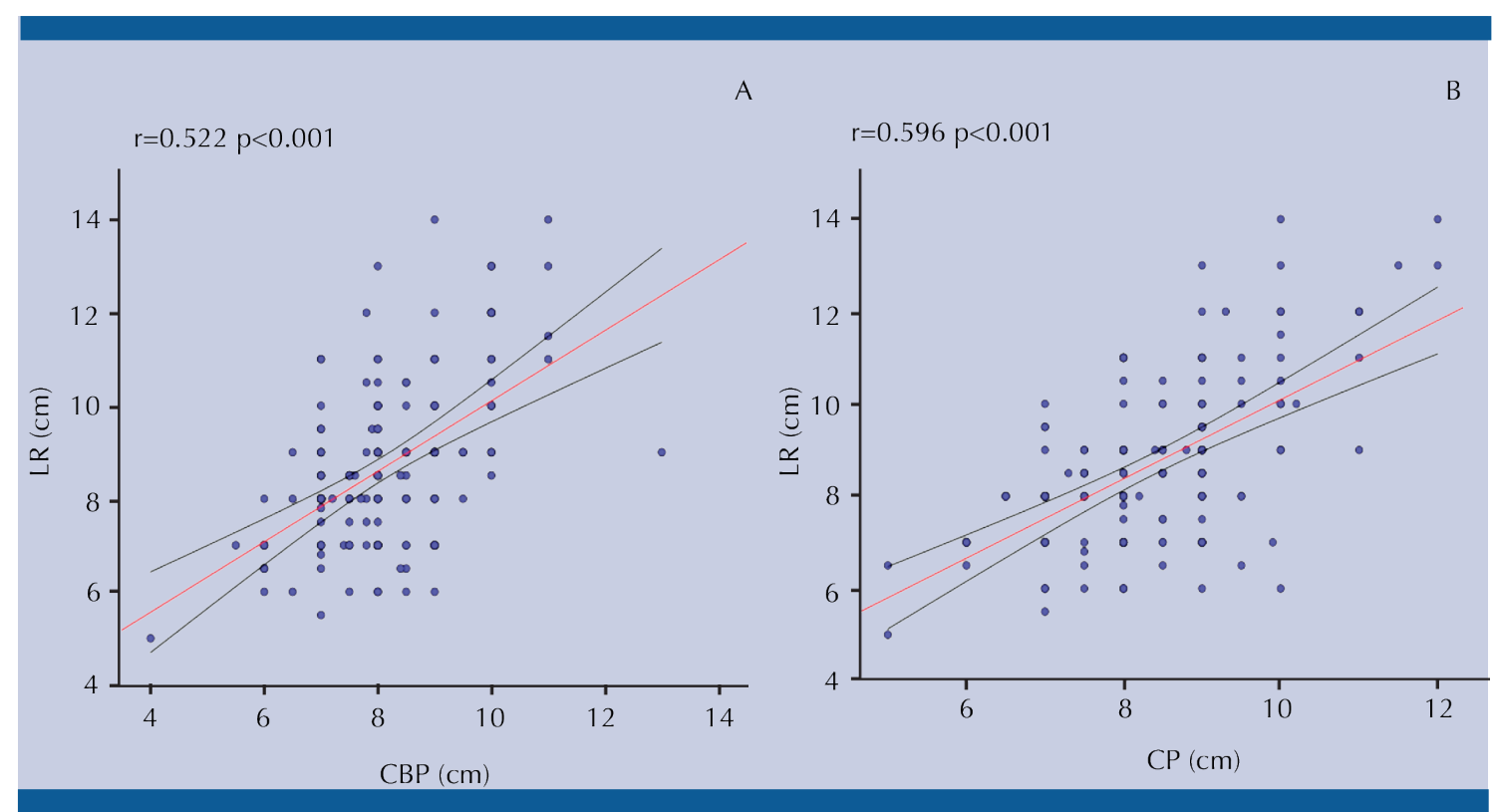

Figura 3. Correlación lineal positiva de la longitud del pene con su circunferencia.

LR: longitud del pene en reposo; CBP: circunferencia de la base del pene; CP: circunferencia del surco balano-prepucial.

cm; al examinar el estado del pene en erección obtuvieron una longitud de $13 \mathrm{~cm}$ y circunferencia de $11.46 \mathrm{~cm}^{14}$ Solo existe un estudio sudamericano que midió la longitud y el grosor del pene en 50 varones guatemaltecos y entre sus resultados reportaron un tamaño promedio 
Cuadro 3. Correlaciones entre variables antropométricas y medidas peneanas $(n=150)$

\begin{tabular}{|c|c|c|c|c|c|c|c|c|c|c|}
\hline Variables & $\begin{array}{c}\mathrm{LE} \\
(\mathrm{cm})\end{array}$ & $\begin{array}{l}\mathrm{LR} \\
(\mathrm{cm})\end{array}$ & $\begin{array}{l}\text { CBP } \\
(\mathrm{cm})\end{array}$ & $\begin{array}{c}\mathrm{CP} \\
(\mathrm{cm})\end{array}$ & $\begin{array}{c}\text { IMC } \\
\left(\mathrm{kg} / \mathrm{m}^{2}\right)\end{array}$ & $\begin{array}{c}\text { Talla } \\
(\mathbf{m})\end{array}$ & $\begin{array}{l}\text { Peso } \\
\text { (kg) }\end{array}$ & $\begin{array}{c}\mathrm{CA} \\
(\mathrm{cm})\end{array}$ & ICC & $\begin{array}{c}\text { Pliegue } \\
\text { pubiano }(\mathrm{cm})\end{array}$ \\
\hline LE (cm) & & $0.878^{* *}$ & $0.502^{* *}$ & $0.574^{* *}$ & $-0.282^{* *}$ & 0.115 & $-0.204^{*}$ & $-0.197^{*}$ & $-0.221^{*}$ & -0.044 \\
\hline LR $(\mathrm{cm})$ & & & $0.522^{* *}$ & $0.596^{* *}$ & $-0.235^{* *}$ & 0.096 & $-0.167^{*}$ & -0.120 & -0.168 & 0.019 \\
\hline CBP $(\mathrm{cm})$ & & & & $0.660^{* *}$ & -0.123 & $0.212^{* *}$ & -0.001 & -0.055 & -0.059 & -0.106 \\
\hline $\mathrm{CP}(\mathrm{cm})$ & & & & & -0.135 & $0.193^{*}$ & -0.026 & 0.009 & -0.015 & -0.057 \\
\hline IMC $\left(\mathrm{kg} / \mathrm{m}^{2}\right)$ & & & & & & -0.064 & $0.866^{* *}$ & $0.678^{* *}$ & $0.429^{* *}$ & 0.135 \\
\hline Talla (m) & & & & & & & $0.439^{* *}$ & 0.085 & -0.048 & -0.076 \\
\hline Peso (kg) & & & & & & & & $0.653^{* *}$ & $0.378^{* *}$ & 0.082 \\
\hline $\mathrm{CA}(\mathrm{cm})$ & & & & & & & & & $0.691^{* *}$ & 0.082 \\
\hline ICC & & & & & & & & & & 0.038 \\
\hline $\begin{array}{l}\text { Pliegue } \\
\text { pubiano }(\mathrm{cm})\end{array}$ & & & & & & & & & & 1 \\
\hline $\begin{array}{l}\text { Correlaciones pos } \\
\text { Correlaciones neg } \\
{ }^{* *} \text { Correlación es } \\
\text { * Correlación esta } \\
\text { IMC: índice de m }\end{array}$ & $\begin{array}{l}\text { tivas: LF } \\
\text { ativas: } \mathrm{L} \\
\text { adístican } \\
\text { dísticam }\end{array}$ & $\begin{array}{l}\text { R con CP y } \\
\text { E con IMC, } \\
\text { mente signif } \\
\text { mente signifi }\end{array}$ & $\begin{array}{l}\text { CBP, y CBP } \\
\text { ICC y peso } \\
\text { ficativa con } \\
\text { cativa con } p \\
\text { dice cintura }\end{array}$ & $\begin{array}{l}\text { con talla. } \\
\mathrm{p}<0.01 \\
<0.05\end{array}$ & & & 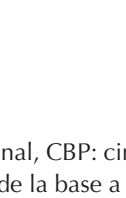 & 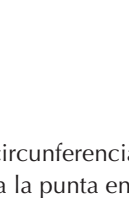 & & ene, CP: circur \\
\hline
\end{tabular}

de $10 \mathrm{~cm}$ y grosor de $8 \mathrm{~cm}$, ambos en reposo, sin encontrar diferencias significativas en la edad. ${ }^{9}$ La revisión sistemática más grande publicada incluyó 15,521 hombres y entre las variables analizadas reportó una longitud promedio en flacidez de $9.16 \mathrm{~cm}$ y alargado de $13.24 \mathrm{~cm}$, con longitud en erección de 13.2, circunferencia en reposo de $9 \mathrm{~cm}$ y en erección de $11.66 \mathrm{~cm}$ (Cuadro 4). ${ }^{10,11,13-15}$

Pocos estudios han evaluado la relación entre la longitud del pene y los parámetros somatométricos. El ensayo de Shah no informa ninguna correlación entre la talla o tamaño del pie con la longitud del pene. ${ }^{16}$ Ponchietti y su grupo encontraron una correlación positiva entre los parámetros somatométricos (altura, peso e IMC) y la longitud del pene. ${ }^{13}$ Un estudio efectuado en Turquía investigó la correlación entre el tamaño del pene y las medidas antropométricas en 1132 personas. Entre sus resultados señalaron que la longitud media del pene en estados flácidos y estirados fue de $9.3 \pm 1.3$ vs $13.7 \pm 1.6 \mathrm{~cm}$, respectivamente ( $p<0.001)$, lo que sugirió una correlación positiva entre la longitud del pene flácido y estirado $(r=0.800, P<0.001)$. Además, encontraron correlaciones leves entre la longitud del pene (flácido y estirado) y la altura, el peso e IMC $\left(p<0.001\right.$ para todos). ${ }^{17}$

Una encuesta realizada en Internet demostró que el tamaño del pene se correlaciona positivamente con la estatura y negativamente con la grasa corporal. ${ }^{18}$ Otro estudio reportó una correlación significativa entre la edad, altura, longitud del dedo índice y las dimensiones del pene, pero no encontraron relación significativa con el índice cintura-cadera y el peso. ${ }^{19}$ En el estudio aquí realizado no encontramos correlación con la talla; sin embargo, se observó una correlación negativa con el IMC e índice cintura-cadera.

En nuestra serie, $22(14.7 \%)$ pacientes percibieron el tamaño del pene inferior al normal; 
Carrillo-Córdova LD. Medidas antropométricas y peneanas

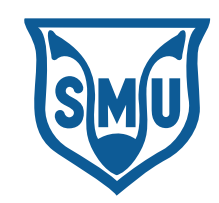

Cuadro 4. Medidas de longitud y diámetro del pene en distintas series ${ }^{10,11,13-15}$

\begin{tabular}{|l|c|c|c|c|c|c|c|c|}
\hline Autor & Año & $\mathbf{n}$ & Edad & $\begin{array}{c}\text { Longitud } \\
\text { flácido }\end{array}$ & $\begin{array}{c}\text { Longitud } \\
\text { estirado }\end{array}$ & $\begin{array}{c}\text { Longitud } \\
\text { erecto }\end{array}$ & $\begin{array}{c}\text { Diámetro } \\
\text { flácido }\end{array}$ & $\begin{array}{c}\text { Diámetro } \\
\text { erecto }\end{array}$ \\
\hline Loeb & 1899 & 50 & $17-35$ & 9.41 & - & - & - & - \\
\hline Schonfeld & 1942 & 196 & $17-25$ & - & 13.1 & - & 8.5 & 15.8 \\
\hline Kinsey & 1948 & 2770 & $20-59$ & 9.7 & 16.74 & - & - & - \\
\hline Bondil & 1992 & 905 & $17-91$ & 10.7 & 16.74 & - & - & - \\
\hline De Ros & 1994 & 150 & - & - & - & 14.5 & 11.5 & - \\
\hline Wessells & 1996 & 80 & $21-82$ & 8.85 & 12.45 & 12.89 & 9.71 & 12.30 \\
\hline Bogaert & 1999 & 935 & - & 10.41 & - & 16.4 & 9.75 & 12.57 \\
\hline Ponchietti & 2001 & 3300 & $17-19$ & 9 & 12.5 & - & 10 & - \\
\hline Schneider & 2001 & 111 & $18-32$ & 8.6 & - & 14.48 & - & - \\
\hline Awwad & 2005 & 271 & $17-83$ & 9.3 & 13.5 & - & 8.9 & - \\
\hline Herbenick & 2015 & 365 & - & - & - & 14.15 & - & 12.23 \\
\hline Veale & 2015 & 15521 & - & 9.16 & 13.24 & 13.2 & 9 & 11.66 \\
\hline
\end{tabular}

no obstante, al compararlos con la población general se encontró que estaban dentro de los parámetros de referencia. En un estudio italiano, $44(65.7 \%)$ adultos jóvenes refirieron un pene corto flácido, $22(32.8 \%)$ flácido y erecto, y 57 (85\%) pensaron que la longitud normal del pene debería ser de 10 a $17 \mathrm{~cm}$. No encontraron ningún paciente con longitud del pene por debajo del percentil 2.5 según su nomograma. Cuarenta y dos $(62.7 \%)$ sujetos mencionaron su problema desde la infancia y 25 (37.3\%) indicaron iniciar en la adolescencia, después de observar imágenes eróticas. Concluyeron que la mayoría de los hombres que solicita la cirugía de alargamiento del pene sobrestima la longitud "normal" del pene. ${ }^{13}$

\section{CONCLUSIONES}

El tamaño promedio del pene en la población mexicana es similar al reportado en otras series internacionales. La talla no se correlaciona con las mediciones del pene; sin embargo, existe correlación lineal negativa con el IMC e índice cintura-cadera, y positiva con la circunferencia peneana. El grado de conformidad o satisfacción del tamaño del pene tiene deficiente concordancia con encontrarse por debajo de la media poblacional.

\section{REFERENCIAS}

1. Schonfeld W. Primary and secondary sexual characteristics study of their development in males from birth through maturity, with biometric study of penis and testes. doi:10.1001/archpedi.1943.02010160019003

2. Fisher WA, et al. The bigger the better? arousal and attributional responses to erotic stimuli that depict different size penises. https://doi.org/10.1080/00224498309551199

3. Ferreira dos Reis M de M, et al. Perceptions about penis size among supposedly healthy 40 to 60 -year-old Brazilian men: a cross-sectional pilot study. http://dx.doi. org/10.1590/1516-3180.2013.7710008

4. Veale $D$, et al. Relationship between self-discrepancy and worries about penis size in men with body dysmorphic disorder. https://doi.org/10.1016/j.bodyim.2016.02.004

5. Guzman-Esquivel J. Micropene. Rev Mex Urol 2008;68(1):1-2.

6. Schonfeld WA, et al. Normal growth and variation in the male genitalia from birth to maturity. J Urol. DOI: https:// doi.org/10.1016/S0022-5347(17)70767-7

7. Sparling J. Penile erections: Shape, angle, and length. https://doi.org/10.1080/00926239708403924

8. Lee PA. Survey report: Concept of penis size. https://doi. org/10.1080/00926239608404917 
9. Veale $D$, et al. Am i normal? A systematic review and construction of nomograms for flaccid and erect penis length and circumference in up to 15521 men. https:// doi.org/10.1111/bju.13010

10. Herbenick D, et al. Erect Penile Length and Circumference Dimensions of 1,661 Sexually Active Men in the United States. DOI: https://doi.org/10.1111/jsm.12244

11. Habous $\mathrm{M}$, et al. Erect Penile Dimensions in a Cohort of 778 Middle Eastern Men: Establishment of a Nomogram. DOI: https://doi.org/10.1111/jsm.12894

12. Granados-Loarca EA, et al. Longitud y grosor del pene. Rev Int Androl. 2005;3(3):109-111.

13. Ponchietti $R$, et al. Penile length and circumference: A study on 3,300 young Italian males. https://doi. org/10.1159/000052434
14. Promodu K, et al. Penile length and circumference: An Indian study. Int J Impot Res. 2007;19(6):558-563.

15. Munding MD, Wessells HB, Dalkin BL. Pilot study of changes in stretched penile length 3 months after radical retropubic prostatectomy. Adult Urol 2001;54(4):568-569.

16. Shah J, et al. Can shoe size predict penile length? BJU Int. 2002;90(6):586-587.

17. Aslan $\mathrm{Y}$, et al. Penile length and somatometric parameters: a study in healthy young Turkish men. Asian J Androl 2011;13(2):339-341.

18. Lever J, et al. Does size matter? Men's and women's views on penis size across the lifespan. http://dx.doi. org/10.1037/1524-9220.7.3.129

19. Mehraban D, et al. Penile size and somatometric parameters among Iranian normal adult men. https://doi. org/10.1038/sj.ijir.3901532

\section{AVISO IMPORTANTE}

La Revista Mexicana de Urología se convierte en una publicación solo digital, con todas las ventajas que los medios y dispositivos electrónicos ofrecen. Usted podrá revisar la información mediante el sitio web (www.revistamexicanadeurologia.org.mx) o descargando la app para Android o iPhone.

Para consultar el texto completo de los artículos deberá registrarse por una sola vez con su correo electrónico, crear una contraseña, indicar su nombre, apellidos y especialidad.

Esta información es indispensable para saber qué consulta y cuáles son sus intereses, y poder en el futuro inmediato satisfacer sus necesidades de información. 